\title{
ON A $p$-ADIC VANISHING THEOREM OF GARLAND
}

\author{
BY W. CASSELMAN ${ }^{1}$
}

Communicated April 15, 1974

Let $k$ be any nonarchimedean locally compact field, with (say) $q$ elements in its residue field. Let $\boldsymbol{G}$ be a simple, semisimple algebraic group defined over $k, G$ the group of its $k$-rational points on $\boldsymbol{G}$. Let $l$ be the $k$-rank of $\boldsymbol{G}$. Suppose $\Gamma$ to be a discrete subgroup of $G$ such that $\Gamma \backslash G$ is compact, and $V$ a finite-dimensional vector space over $C$ on which $\Gamma$ has a unitary representation. In [4], Garland has proven that there exists an integer $q(l)$ (depending only on $l$ ) such that if $q>q(l)$ then $H^{m}(\Gamma, V)=0$ for $m \neq 0, l$. Garland's proof is an analogue of the proofs of vanishing theorems for discrete subgroups of real groups, applying a sort of curvature on the Bruhat-Tits complex of $G$. By an apparently entirely different method, I have been able to remove the residue field restriction and thus to prove

Theorem 1. One has $H^{m}(\Gamma, V)=0$ for $m \neq 0, l$.

The proof involves the continuous cohomology and the theory of admissible representations of $G$. Let me show how these enter into consideration.

First of all, one may assume $\boldsymbol{G}$ to be simply connected (see Lemma 3.4 in [1]). Let

$$
I_{V}=\operatorname{Ind}(V \mid \Gamma, G)
$$

be the space of all locally constant functions $f: G \rightarrow V$ such that $f(\gamma g)=$ $\gamma \cdot f(g)$ for all $\gamma \in \Gamma, g \in G$. The group $G$ acts on this by right translation: $R_{g} f(x)=f(x g)$. With this action, since $\Gamma \mid G$ is compact, the space $I_{V}$ is that of an admissible representation of $G$, i.e., every element in this space is fixed by some compact open subgroup of $G$, and for any compact open subgroup $K$, the space $I_{V}^{K}=\left\{f \in I_{V} \mid R(k) f=f\right.$ for all $\left.k \in K\right\}$ has finite dimension. Since $V$ is a unitary $\Gamma$-module and there exists a $G$-invariant measure on $\Gamma \backslash G$, the representation of $G$ on $I_{V}$ is unitary as well. Admissibility and unitarity together of $I_{V}$ imply easily that it is $G$-isomorphic to a direct sum $\oplus I_{n}$ of irreducible, admissible, unitary $G$-spaces, each isomorphism class occurring with finite multiplicity.

AMS (MOS) subject classifications (1970). Primary 20E40, 20G10, $20 \mathrm{G} 25$.

${ }^{1}$ The author has been partially supported at the Institute for Advanced Study by NSF grant GP36418X1 while finishing the research described here. 
It follows, then, from [3, Proposition 3 and Corollary 3 to Theorem 2] that

$$
H^{*}(\Gamma, V) \cong H_{\mathrm{cont}}^{*}\left(G, I_{V}\right) \cong \bigoplus H_{\mathrm{cont}}^{*}\left(G, I_{n}\right),
$$

where $H_{\text {cont }}^{*}$ means continuous cohomology. (Any admissible representation is continuous with respect to the discrete topology on the vector space, and that is the topology implied here.) One, therefore, comes to the question: for which irreducible unitary admissible $G$-representation spaces $I$ is $H_{\text {cont }}^{*}(G, I) \neq 0$ ?

Let $P_{\varnothing}$ be (the $k$-rational points of) a minimal parabolic subgroup of $G, A_{\varnothing}$ a maximal split torus in $P_{\varnothing}, \Delta$ the corresponding set of simple positive roots. For each subset $\Theta \subseteq \Delta$, let $P_{\Theta}$ be the corresponding standard parabolic subgroup (so that $P_{\varnothing}$ is named correctly, and $P_{\Delta}=G$ ). For every such $\Theta$, let $\pi_{\Theta}$ be the right regular representation of $G$ on the space

$$
V_{\Theta}=C^{\infty}\left(P_{\Theta} \mid G\right)=\left\{\text { locally constant } f: P_{\Theta} \mid G \rightarrow C\right\} .
$$

This defines an admissible representation of $G$. For $\Theta \subseteq \Omega$, one has $\pi_{\Omega} \subseteq \pi_{\Theta}$. Define $\sigma_{\Theta}$ to be the representation of $G$ on $U_{\Theta}=V_{\Theta} / \Sigma_{\Theta \subsetneq \Omega} V_{\Omega}$. Thus, $\sigma_{\Delta}$ is the trivial representation of $G$, and $\sigma_{\varnothing}$ is the Steinberg representation of $G$ (see [2]).

THEOREM 2. (a) Each $\sigma_{\Theta}$ is an irreducible representation.

(b) For each $\Theta$,

$$
\begin{aligned}
H_{\text {cont }}^{m}\left(G, V_{\Theta}\right) & =0 \quad \text { if } m \neq|\Delta-\Theta|, \\
& \cong C \quad \text { if } m=|\Delta-\Theta| .
\end{aligned}
$$

(c) If $V$ is the space of any irreducible admissible representation of $G$, then $H_{\text {cont }}^{*}(G, V)=0$ unless $V$ is G-isomorphic to one of the $V_{\Theta}$.

(d) The only representations $\sigma_{\Theta}$ which are unitary are $\sigma_{\varnothing}$ and $\sigma_{\Delta}$.

I shall not even sketch the proof of this theorem, which is difficult and involves a great deal of the theory of admissible representations of $G$. It is part (d) which is subtle; this was first proved by A. Borel by methods quite similar to Garland's proof of Theorem 1, but under a similar residue field restriction! (See $[1, \S 2.1]$.)

From Theorem 2 to Theorem 1 is a simple step. It follows from (a), (b), (c), and earlier remarks that

$$
H^{m}(\Gamma, V) \cong \underset{|\Theta|=l-m}{\bigoplus} \operatorname{Hom}_{G}\left(V_{\Theta}, I_{V}\right)
$$

But since $I_{V}$ is unitary, (d) implies that $\operatorname{Hom}_{G}\left(V_{\Theta}, I_{V}\right)=0$ unless $\Theta=\varnothing$ or $\Delta$. And for the case $\Theta=\varnothing$, one obtains a result complementary to but 
more elementary than Theorem 1 (known already to Garland, and proved independently by Borel and Serre):

THEOREM 3. If $\boldsymbol{G}$ is simply connected, then $H^{l}(\Gamma, V) \cong \operatorname{Hom}_{G}\left(V_{\varnothing}, I_{V}\right)$.

Now assume $\left\{k_{i}\right\}$ to be a finite set of nonarchimedean local fields. For each $i$, let $\boldsymbol{G}_{i}$ be a simple, semisimple algebraic group defined over $k_{i}$, let $l_{i}$ be the $k_{i}$-rank of $\boldsymbol{G}_{i}$, and let $G_{i}$ be the group of $k_{i}$-rational points of $\boldsymbol{G}_{i}$. Let $G$ be $\prod G_{i}, l=\sum l_{i}, \Gamma$ a discrete subgroup of $G$ such that (i) $\Gamma \backslash G$ is compact, and (ii) the projection of $\Gamma$ onto each factor $G_{\hat{i}}=$ $\prod_{j \neq i} G_{j}$ is dense. Let $V$ be a finite-dimensional unitary $\Gamma$-module.

Theorem 4. One has $H^{m}(\Gamma, V)=0$ unless $m=0, l$.

This was first proved by Borel (see [1, $§ 6.5]$ ), again under a residue field restriction for each factor. Of course, one may generalize it slightly to include the case, for example, where $\boldsymbol{G}$ is any semisimple group over a single field. The proof of this result again depends on Theorem 2, and follows closely that of Theorem 1 . One needs along the way three elementary results.

Lemma 1. Any irreducible admissible representation $\pi$ of $\prod G_{i}$ is a tensor product $\otimes \pi_{i}$ of irreducible admissible representations of the factors $G_{i}$.

LEMMA 2. If $\pi=\otimes \pi_{i}$ is a tensor product of irreducible representations of $G$, one of which is trivial, then $\operatorname{Hom}_{\Gamma}(\pi, V)=0$ unless $\pi \cong C$.

LEMMA 3. If $\pi$ is an irreducible representation of $G$ such that $\operatorname{Hom}_{G}\left(\pi, I_{V}\right) \neq 0$, then $\operatorname{Hom}_{\Gamma}(\pi, V) \neq 0$.

Let me point out that (1) the results of this paper for the vanishing of one-dimensional cohomology were originally proved by Kazhdan in [5], who also used the representation theory for $G$, but in a rather different way; and (2) the vanishing theorems were originally conjectured by Serre in [6]. I would like to raise the point that Serre's conjecture actually involved representations of $\Gamma$ on $k$-vector spaces, inherited from a rational representation of $\boldsymbol{G}$. A number of cases of this are also covered by the above results (see $[4, \S 9]$ ), but for both Garland's techniques and mine the assumption of complex coefficients, and of unitarity, is crucial. A reasonable theory of infinite-dimensional $p$-adic representations of a $p$-adic group does not yet exist, for example.

One interesting question is to what extent one can pursue the methods suggested here for discrete subgroups of real Lie groups. It may not be a reasonable task, because one knows already in this case that the $\sigma_{\Theta}$ 's of Theorem 2 generally have no simple parallel. 
I would finally like to mention that Garland himself, according to a conversation in February, 1974, has been able to remove the residue field restriction in a large number of cases, by a refinement of the calculations in [4].

\section{REFERENCES}

1. A. Borel, Cohomologie de certains groupes discrets et Laplacien p-adique, Séminaire Bourbaki: 1973, Exposé 437.

2. W. Casselman, The Steinberg character as a true character, Proc. Sympos. Pure Math., vol. 26, Amer. Math. Soc., Providence, R.I., 1972, pp. 413-418.

3. W. Casselman and D. Wigner, Continuous cohomology and a conjecture of Serre's, Invent. Math. (to appear).

4. H. Garland, p-adic curvature and the cohomology of discrete subgroups, Ann. of Math. (2) 97 (1973), 375-423.

5. D. A. Každan, Connection of the dual space of a group with the structure of its closed subgroups, Funkcional Anal. i Priložen. 1 (1967), 71-74=Functional Anal. Appl. 1 (1967), 63-65. MR 35 \#288.

6. J.-P. Serre, Cohomologie des groupes discrets, Prospects in Mathematics, Ann. of Math. Studies, no. 70, Princeton Univ. Press, Princeton, N.J., 1970.

Department of Mathematics, University of British Columbia, Vancouver, British Columbia, Canada 